\title{
CARACTERIZACIÓN TÉCNICA Y SOCIOECONÓMICA DE LA PRODUCCIÓN OVINA EN EL ESTADO DE YUCATÁN, MÉXICO ${ }^{1}$
}

\author{
Rubén Dario Góngora-Pérez², Sergio Fernando Góngora-González $z^{3}$, Miguel Ángel Magaña-Magaña ${ }^{4}$ \\ Pedro Enrique Lara y Lara $^{4}$
}

\section{RESUMEN}

Caracterización técnica y socioeconómica de la producción ovina en el estado de Yucatán, México. El objetivo de este trabajo fue determinar la situación socioeconómica de la producción ovina en el estado de Yucatán México, durante el año 2007. El método de investigación consistió en el levantamiento de encuestas mediante cédulas de entrevista, estratificando las unidades de producción (UP) por número de vientres (E1 de 1-20, E2 de 21-50 y E3 de más de 50). Las fuentes alimenticias son praderas, alimento concentrado, agostaderos y pasturas de corte, y se suplementa con minerales. El empadre continuo $(76,7 \%)$ es común, la inseminación artificial se realiza solo en E3 (2,2\%). Las enfermedades frecuentes y con mayor incidencia en E1 son: la neumonía (37\%), clostridio $(27,7 \%)$ y el carbón sintomático $(20,2 \%)$. Los períodos de desparasitación se dan fuera de lo recomendado: cada seis meses $(41,7 \%)$ y anual $(33,8 \%)$. El cuidado de la hembra próxima al parto y del cordero, se realizan en un $63,3 \%$, y aumenta según el estrato; mientras que los abortos disminuyen al aumentar el estrato. La mortalidad presentó índices normales, solamente en E1, la mortalidad predestete fue elevada $(12,2 \%)$. Los principales criterios para la venta de animales fueron: peso del animal y necesidad de dinero, y ésta se realizó comúnmente en la UP $(83,7 \%)$. Los gastos de mayor magnitud son: mano de obra $(23,7 \%)$ y alimentación $(21,9 \%)$. El ingreso proviene de la venta de animales con peso al mercado y destetes; la rentabilidad sólo fue positiva para el estrato 3, con ganancias promedio de $\$ 2,8$ por kilogramo de carne en pie.

Palabras claves: Ovino, comercialización, costos, rentabilidad.

\begin{abstract}
Technical and socioeconomic characterization of the ovine production in the state of Yucatan, México. The objective was to determine the socioeconomic status of sheep production in the state of Yucatán Mexico, during year 2007. The investigation protocol consisted on the application of surveys through interviews forms, stratifying the production units (PU) by the number of wombs (E1 from 1-20, E2 from 21-50, E3 more than 50). The feed sources were grassland, concentrated feed, rangeland and cut forage supplemented with minerals. Continuous coupling (76.7\%) was common, and artificial insemination is used only in E3 $(2.2 \%)$. Frequent diseases and with higher incidence in E1 were pneumonia (37\%), clostridium (27.7\%), and blackleg (20.2\%) Use of anti-parasites is out of time, each 6 months $(41.7 \%)$ and each year $(33.8 \%)$. Care tacking of the pregnant sheep and lambs is conducted in a $63.3 \%$ and increases according to the number of wombs. Mortality showed standard levels only in E, and the level of mortality was high during the nurturing period $(12.2 \%)$. The main criteria to sell the animals were: animal weight and cash needs, and sales usually took place at the PU (83.7\%). The highest costs are labor (workforce) $(23.7 \%)$ and feeding (21.9\%). Income comes from the sale of animals with market weight and in post-weaning stage; profitability was positive only for E3, with standard earnings of $\$ 2.8$ per Kilo of standing meat.
\end{abstract}

Key words: Sheep, commercialization, costs, yield.

\footnotetext{
Recibido: 21 agosto, 2009. Aceptado: 24 de mayo, 2010. Proyecto financiado por el Fondo Sectorial de Investigación SAGARPACONACYT. Clave del proyecto: SAGARPA-2004-C01-150, siendo sujeto del apoyo: INIFAP.

2 Red Nacional de Innovación en Socioeconomía. Campo Experimental Chetumal CIR-SURESTE. Dirección: Km 25 carretera ChetumalBacalar C.P. 77000, Quintana Roo, México. Tel (983) 8320167. gongora.ruben@inifap.gob.mx

3 Red Nacional de Innovación en Socioeconomía. Campo Experimental Mococha, CIRSE-INIFAP. Dirección: Calle 6 \# 398 X 13. Avenida: Correa Rachó, Colonia: Díaz Ordaz, C.P. 97130, Mérida Yucatán, México. Tel. Fax. (999) 1961182 ext. 302. gongora.sergio@inifap.gob.mx

4 División de Estudios de Posgrado e Investigación, Instituto Tecnológico de Conkal, Yucatán, México. Dirección: Km. 16.3 antigua carretera Mérida - Motul, Conkal, Yucatán. Tel. Fax: 01 (999) 912-41-30, 912-41-35, ext 122 y 121.drmmagana@gmail.com; plara@itaconkal.edu.mx
} 


\section{INTRODUCCIÓN}

Las actividades pecuarias mantienen una gran importancia en el contexto socioeconómico del país y al igual que el resto del sector primario, han servido de base al desarrollo de la industria nacional. La producción de carne ovina es la actividad productiva más diseminada en el medio rural; se realiza sin excepción en todas las regiones ecológicas del país y aún en condiciones adversas de clima, que no permiten la práctica de otras actividades productivas.

La producción ovina en México, inició un proceso de diversificación a partir de la década de los años noventa. La consecuencia de ésta fue un aumento del inventario animal, el cual paso de seis a casi nueve millones de cabezas entre 1996 y 2006; este incremento se debió, entre otros factores, al continuo aumento del precio del ovino en pie, al pasar de $\$ 5,98$ a $\$ 20,47$ en el periodo de 1994 a 2004, respectivamente (SIAP SAGARPA 2006).

La producción ovina se ha extendido a gran parte de la región tropical de México. El estado de Yucatán, la ovinocultura en la zona centro-norte, era una actividad que se practicó de manera tradicional, con rebaños explotados en su mayoría a nivel de traspatio, con infraestructura rústica y sistemas de alimentación poco definidos, lo cual se traducía en índices de productividad relativamente bajos (Franco et al. 1992). Actualmente el panorama de la ovinocultura es diferente en el estado de Yucatán, ya que presenta cambios sustanciales como la conformación de asociaciones especializadas en ovinos, mercado constante, precios atractivos y apoyos gubernamentales, reflejando la inclusión de nuevos productores con inversiones importantes, lo que permite en mayor o menor medida la incorporación de prácticas tecnológicas en el manejo del rebaño, con finalidades de producción definidas. Estudios en sistemas de producción ovina basados en la raza Pelibuey en Yucatán, indican que de acuerdo al tipo de productor (asociado o no) se refleja el tamaño del rebaño, grado de tecnificación, y por consiguiente su proceso de comercialización (Magaña et al. 2000).

Dado el dinamismo y cambios de la producción ovina, así como el papel de la investigación en México, el cual se ha caracterizado por generar tecnologías sin tomar en consideración la tipología de los productores, o bien impulsando tecnologías para aquellos productores que cuentan con los recursos económicos para aplicarlas y cuyo principal objetivo es incrementar sus rendimientos y ganancias, sin tomar en cuenta a los productores de escasos recursos. El presente trabajo tuvo como objetivo determinar la situación socioeconómica de la producción ovina en el estado de Yucatán México, durante el 2007.

\section{MATERIALES Y MÉTODOS}

El estado de Yucatán, región de estudio de este proyecto, se extiende en una superficie de $39340 \mathrm{~km}^{2}$ entre los paralelos $190^{\circ} 35^{\prime}$ y $210^{\circ} 35^{\prime}$ de latitud norte y los meridianos $870^{\circ} 30^{\prime}$ y $900^{\circ} 15^{\prime}$ de longitud oeste (INEGI 2006). Limita al Norte con el Golfo de México, al Este con el territorio de Quintana Roo y al Oeste con el estado de Campeche. El clima dominante es de tipo tropical húmedo, con una temperatura media anual de $25,90{ }^{\circ} \mathrm{C}$ y precipitación pluvial media anual desde los $500 \mathrm{~mm}$ hasta los $1500 \mathrm{~mm}$ (CONAGUA 2008).

Los principales ecosistemas son la selva baja caducifolia del tipo secundario en la parte Suroeste y en los extremos Centro-Norte. También existe una mínima extensión de selva baja perennifolia. El uso del suelo es principalmente agrícola, el cultivo más importante es el henequén, pero también se produce maíz, frijol, chile, calabaza y naranja.

El estudio se elaboró en el año 2007, con base en los siguientes pasos: a) Elaboración de la cédula de entrevista, estructurada con 10 secciones, fue sometida a un proceso de validación con expertos investigadores por área de conocimiento y una validación en campo con 10 productores para determinar su funcionalidad al llenado, b) Obtención de la base de datos de los productores, con base en el padrón de los cuatro distritos de desarrollo rural del estado, c) Levantamiento de la información, ésta se llevó a cabo de manera personal con el dueño de la unidad de producción y en algunos casos en el mismo rancho, d) Sistematización de la información, ésta se incluyó en una base de datos del programa Excel, e) El tamaño de muestra, la estratificación y el análisis de datos se llevaron a cabo por los siguientes métodos:

Para determinar la muestra de productores, se utilizó el Muestreo Aleatorio Estratificado con Distribución Proporcional, mediante la siguiente fórmula:

$$
n=\frac{N \sum N i D e i^{2}}{N^{2} D^{2}+\sum N i D i^{2}}
$$

AGRONOMÍA MESOAMERICANA 21(1):131-144. 2010 
$\mathrm{n}=$ Tamaño de la muestra

$\mathrm{N}=$ Tamaño de la población

$\mathrm{Ni}=$ Número de individuos del estrato " $\mathrm{i}$ "

$\mathrm{Dei}^{2}=$ Varianza del estrato " $\mathrm{i}$ "

$\mathrm{D}=\mathrm{d} / \mathrm{z}$

$\mathrm{d}=$ Precisión deseada

$\mathrm{z}=$ Valor crítico de la distribución $\mathrm{z}$

La estratificación se realizó considerando el número de vientres por productor y la distribución de la muestra por estrato:

$$
n i=n{ }_{N}^{N i}
$$

ni = Tamaño de muestra en el estrato "i"

$\mathrm{Ni}=$ Número de individuos del estrato "i"

$\mathrm{N}=$ Tamaño de la población

La muestra final de productores quedó conformada como se presenta en el Cuadro 1.

Cuadro 1. Muestra y estratificación de productores considerando el numero de vientres, en el estado de Yucatán, México. 2007

\begin{tabular}{ccc}
\hline Estrato & $\begin{array}{c}\text { Número de } \\
\text { vientres }\end{array}$ & $\begin{array}{c}\text { Número de } \\
\text { productores }\end{array}$ \\
\hline 1 & $1-20$ & 47 \\
2 & $21-50$ & 32 \\
3 & Más de 50 & 11 \\
\hline
\end{tabular}

A partir de la información contenida en las cédulas de entrevista, se elaboró una base de datos en la que se sistematizó el total de variables. El registro correspondiente se revisó con el fin de detectar incongruencias en la información obtenida en campo; los datos no congruentes con la realidad se corrigieron visitando nuevamente al productor, con lo cual se validó la base de datos y garantizó la veracidad de las estimaciones obtenidas de ellas.

\section{Análisis de datos}

\section{Caracterización técnica}

Los principales indicadores se estimaron por medio de las rutinas de cálculo, con apoyo del programa
Microsoft Office Excel. Se obtuvieron las estadísticas descriptivas para identificar y caracterizar el proceso de producción ovina con base en las prácticas tecnológicas y de manejo de los rebaños.

\section{Análisis de comercialización}

La información del proceso de comercialización se realizó con el programa Microsoft Office Excel, mediante estadística descriptiva.

\section{Estudio socioeconómico}

De la base de datos generada se sistematizó el total de las variables, las cuales se sometieron a un análisis de varianza por medio del programa Statistix software, comparando las medias por Scheffe (Murray y Larry 2001) y la correlación de Pearson para las variables escolaridad, experiencia en la producción de ovinos (años), superficie (ha) y productividad (kg corderos producidos por vientre).

\section{Análisis de costos y rentabilidad}

Para la determinación del Costo Variable Medio (CVM) se consideraron los costos directamente asociados al nivel de producción de la empresa (Wadswoth 1997). Los costos relacionados con la operación diaria del rebaño fueron: alimentación, minerales, desparasitantes, vacunas, control de enfermedades varias, herbicidas, mano de obra, energía eléctrica, mantenimiento de infraestructura y equipo, combustibles y lubricantes.

Para el Costo Fijo Medio (CFM), se consideraron los que no presentan variación en relación con el volumen de producción, basados en estimaciones. Su valor es independiente del nivel de producción del rebaño. Los costos fijos analizados fueron: depreciación de equipo e instalaciones, así como el interés entre inversión de "pie de cría" e infraestructura.

El Costo Medio Total (CMT) se obtuvo de la suma del costo variable medio y del costo fijo medio.

El Ingreso por Ventas de Animales (IVA) se estimó al multiplicar el precio al que se vende el producto por la cantidad del mismo que es enviado al mercado (Kay 1990). Se agregaron los valores obtenidos por la venta de animales con peso al mercado, destetes, vientres, sementales y animales de desecho. La fórmula para la estimación fue: 


$$
I T=\sum_{i=1}^{n} \text { PqixQi }
$$

IT = Ingreso total por ventas

$\mathrm{P}_{\mathrm{qi}}=$ Precio de venta

$\mathrm{Q}_{\mathrm{i}}^{\mathrm{qi}}=$ Cantidad de producto vendida.

La Rentabilidad (П) se estimó al restarle al valor del ingreso total por ventas el valor del costo total, mismo que se forma del costo fijo y costo variable (Kay 1990).

$$
\Pi=I T-C T
$$

IT = Ingreso Total por Ventas

$\mathrm{CT}=$ Costo Total

$\Pi=$ Rentabilidad

\section{RESULTADOS Y DISCUSIÓN}

\section{Características técnicas del proceso de producción}

\section{Manejo nutricional}

Las principales fuentes de alimentación de los rebaños ovinos durante el año fueron: las praderas con gramíneas introducidas, vegetación secundaria y concentrado comercial, proporcionados en diferentes cantidades dependiendo del estrato del productor.

En el estrato 1 (E1) su principal fuente de alimentación fueron las áreas de vegetación secundaria, esto debido a la limitada superficie con la que cuenta el productor para establecer praderas y su bajo nivel económico para realizar dicha inversión. En el estrato 2 (E2) los rebaños pastorean frecuentemente en praderas con gramíneas introducidas, debido a que el área de superficie promedio aumenta y en su mayoría existe inversión de capital para el establecimiento de potreros. Los rebaños en los cuales la actividad se encuentra como una forma de ingreso constante y con capital invertido, se encuentran en el estrato 3 (E3), en estas unidades de producción la principal fuente de alimentación es el alimento concentrado, debido a que en su mayoría se dedican a la engorda de ovinos (Figura 1).

El sistema de alimentación que prevalece para la producción de carne ovina en zonas tropicales está

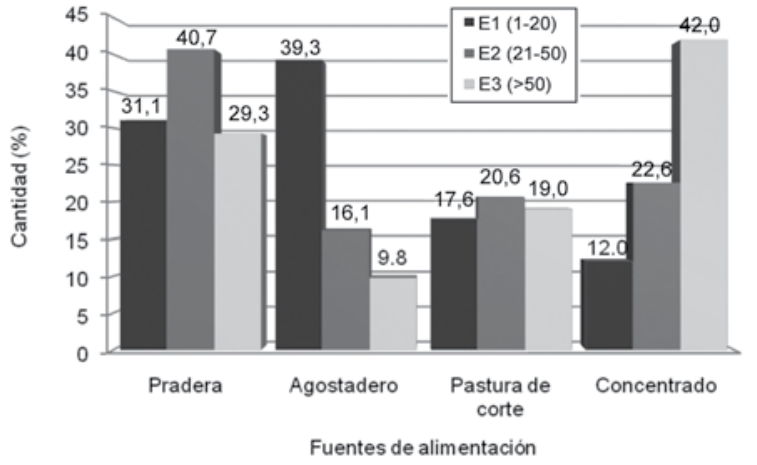

Figura 1. Fuentes de alimentación de los rebaños ovinos y proporcion de uso con base a la cantidad de vientres por unidad de producción en el estado de Yucatán, Mexico. 2007.

basado en el pastoreo con vegetación secundaria o en praderas con gramíneas introducidas, en ambos casos, éstas son las fuentes de alimento más económicas, pero estos recursos son limitados y de bajo valor nutricional en época seca, siendo necesario buscar alternativas de alimentación con el fin de suplementar la dieta de los ovinos. En este análisis la principal fuente de suplementación en época seca para el E1 fue en un 30,4\% el alimento concentrado comercial, no obstante la base de la suplementación para este estrato son el ramón (Brosimun alicastrum) y desechos agroindustriales (pollinaza y esquilmos de cosechas), éstos alcanzan un $36,5 \%$, superior al uso del concentrado. En el E2 el uso del concentrado aumenta al 44,6\%, complementado con el uso de pollinaza y maíz en un 17,9 y $10,7 \%$ respectivamente. En el E3 se observó la mayor frecuencia en el uso de alimento concentrado y minerales, insumos recomendados para la suplementación en época seca, al igual que el uso del zacate de corte del género Pennisetum, ya que en este estrato se encuentra, el mayor número de explotaciones estabuladas, condición que las hace dependientes de estos recursos.

La suplementación que se proporcionaba a los ovinos en su mayoría estuvo destinada a las hembras, en los tres estratos no existió una variación considerable, ya que las hembras son la prioridad en el uso de los suplementos en época de seca $(49,4 \%)$. Esto es similar para sementales y corderos con un 37,7 y $13 \%$ respectivamente, esta práctica dirigida de suplementación se puede explicar por el valor productivo que tienen las hembras para los productores, ya que dichos animales son la base para el desarrollo del rebaño. 
En relación a la suplementacíon mineral se encontró el uso de tres formas de sales que se proporcionan en los estratos estudiados. En el E1 la forma más común en la cual se proporciona la sal es de forma granulada $(44,4 \%)$, seguida del block mineral y de la mezcla de sales con un 37,0 y $18,5 \%$ respectivamente. En el E2, la mezcla de sales es la que más uso tiene con un $50,0 \%$ y la que menos uso tiene es la sal común granulada, con un $12,5 \%$. El block mineral en estrato E3 se adicionó en un 55,6\% y en menor cantidad la sal común granulada, con un $11,1 \%$. El mayor empleo de sal común granulada observada en el E1, se da por su fácil acceso, así como menor precio; en el E3, la principal forma en la que se proporcionaron las sales fue por medio de block de minerales, ésto por lo práctico que resulta su suministro a un mayor número de ovinos dentro del rebaño. En la Figura 2 se muestran las proporciones en la cuales se llevan a cabo la suplementacíon alimenticia, mineral y la elaboración de raciones en los tres estratos de productores.

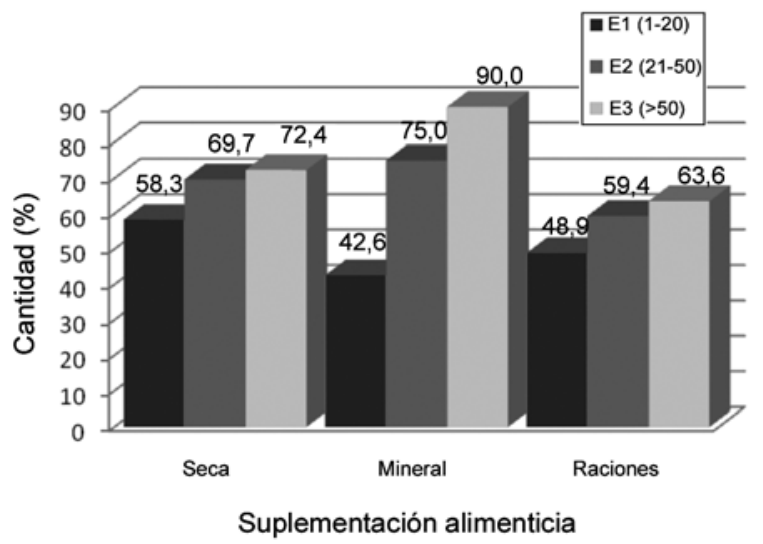

Figura 2. Suplementacion mineral y preparación de raciones para ovinos en época seca, con base en la cantidad de vientres por unidad de producción, en el estado de Yucatan, México. 2007.

\section{Manejo reproductivo y genético}

Los resultados obtenidos en cuanto al manejo reproductivo del rebaño en el estado, indican que el $76,7 \%$ de los productores realizan el empadre continuo, mientras que el $23,3 \%$ de productores restante utiliza al semental solo parte del año o empadre controlado. El análisis por estratos señala que el 80,9\% de los productores del E1 todo el año mantuvo al semental con las hembras, mientras que en el E2 y E3, el 75,0 y $63,3 \%$ lo realizan de la manera señalada con anterioridad. Por el contrario, el estrato que utilizó más al semental mediante un esquema de empadre controlado fue el E3, y en éste se realizó el 36,4\%, seguido de E2 y E1 con 25,0 y 19,1\%, respectivamente.

Por otra parte, las técnicas de reproducción más comunes son la monta directa y la inseminación artificial (I.A.). Estas representaron el 97,8\% para monta directa y $2,2 \%$ para la I.A. Por estratos, la única variante se observó en el E3, donde el 13,6\% de los productores realizan la I.A. mientras que el $86,4 \%$ da montas directas. En el E1 y E2 se utiliza monta directa al 100\% (Figura 3). Las razas comúnmente utilizadas para la I.A. en el estado son la Dorper y Pelibuey.

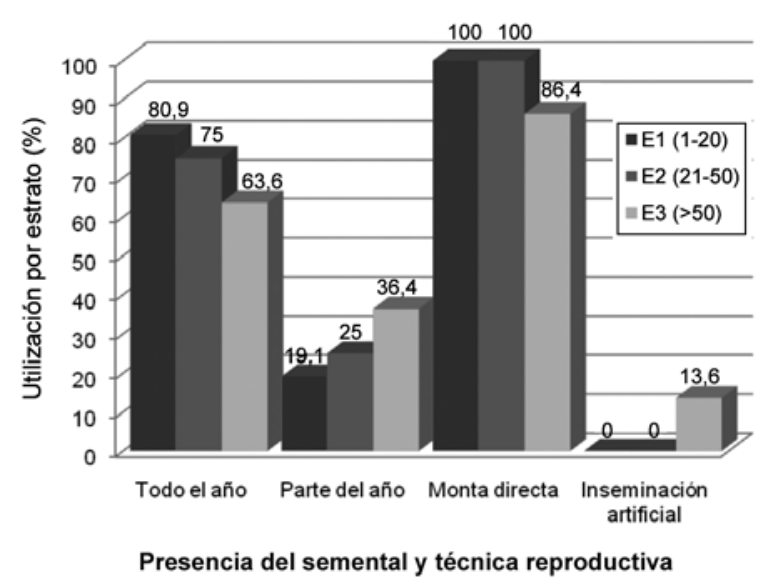

Figura 3. Técnica de reproducción utilizada y permanencia del semental en el rebaño de ovinos, con base en la cantidad de vientres por unidad de producción. Yucatán, México. 2007

En los rebaños del estado solo el 31,3\% cuenta con algún animal puro, mientras que en el $68,7 \%$ restante los animales son producto de cruzas. La incorporación de razas puras a los rebaños ovinos no es una práctica reciente, ha estado a la disposición de solo un tipo de productor; no obstante, los productores con recursos económicos limitados han podido tener acceso a estas razas mediante apoyos gubernamentales. En E1 sólo el 14,9\% tuvo algún animal de raza pura y en E3 aumentó a un 44,5\%. Estos datos reflejan una baja incorporación de animales puros en los rebaños del estado, las razas Pelibuey y Dorper son las más utilizadas (Cuadro 2). 
Cuadro 2. Empleo de razas puras y cruzas en los rebaños ovinos y proporción de uso de las razas puras existentes en el estado de Yucatán, México. 2007.

\begin{tabular}{lcccc}
\hline \multirow{2}{*}{ Razas } & \multicolumn{4}{c}{ Estrato por número de vientres* $\mathbf{( \% )}$} \\
\cline { 2 - 5 } & $\mathbf{E 1}$ & $\mathbf{E 2}$ & $\mathbf{E 3}$ & $\overline{\mathbf{x}}$ \\
\hline Cruzas & 85,1 & 65,1 & 55,5 & 68,7 \\
Puras & 14,9 & 34,4 & 44,5 & 31,3 \\
\hline Pelibuey & 47,8 & 47,6 & 40 & 45,1 \\
Dorper & 21,7 & 33,3 & 50 & 35 \\
Katahdin & 17,4 & 14,3 & 10 & 13,9 \\
Black Belly & 13 & 4,8 & - & 5,9 \\
\hline
\end{tabular}

* Estrato es la unidad de producción de ovinos con base en el número de vientres: $\mathrm{E} 1=1-20 ; \mathrm{E} 2=21-50 ; \mathrm{E} 3=>50$.

Actualmente las cruzas más utilizadas por los productores en el estado, son la de Pelibuey X Black belly y Pelibuey X Dorper con un 47,8 y 23,9\% respectivamente. En orden de importancia, le siguen las cruzas Pelibuey X Katahdin, Black belly X Katahdin y Dorper $\mathrm{X}$ Katahdin con 10,$9 ; 8,7$ y $8,7 \%$ respectivamente. Asimismo, las cruzas terminales más utilizadas son Pelibuey X Black belly X Dorper y Pelibuey X Katahdin $X$ Dorper con 42,9 y $28,6 \%$ respectivamente.

En el estado de Yucatán el problema de consanguinidad no se ha podido evaluar con precisión, sin embargo los productores en la mayoría de los casos lleva a cabo cambio de sementales. El E1 fue el que presentó mayor número de animales emparentados con un $31,9 \%$, seguido por los E2 y E3 con 28,1 y $18,2 \%$ respectivamente. En el caso de los productores que mencionaron no tener problemas de animales emparentados $(71,1 \%)$, realizan el cambio de sementales $(97,2 \%)$ y el $2,8 \%$ restante apartando a sus hembras.

El reemplazo del semental y las hembras se dio con animales del mismo rebaño o bien comprándolos a otros productores. Los sementales se reemplazan frecuentemente por medio de la compra en otros rebaños $(34,4 \%)$, mientras que las hembras se remplazan con otro animal del mismo rebaño $(42,4 \%)$ (Cuadro 3). Los ovinocultores del E3 prefieren comprar sus sementales en otros rebaños $(43,2 \%)$, mientras que en E1 existió mayor reemplazo de sementales del mismo rebaño (21,3\%), para el caso de las hembras, los tres estratos presentaron un porcentaje similar de reemplazos del mismo rebaño con $43,7,41,1$ y $40,5 \%$ respectivamente.
Cuadro 3. Reemplazo y compra de vientres y sementales en los rebaños ovinos del estado de Yucatán México, 2007.

\begin{tabular}{lrrrr}
\hline \multirow{2}{*}{ Reemplazo de animales } & \multicolumn{4}{c}{$\begin{array}{c}\text { Estrato por número de } \\
\text { vientres* }\end{array}$} \\
\cline { 2 - 5 } & \multicolumn{1}{c}{ E1 } & \multicolumn{1}{c}{ E2 } & E3 & Total \\
\hline Sementales comprados & 28,7 & 39,7 & 43,2 & 34,4 \\
Sementales reemplazo & 21,3 & 10,3 & 6,8 & 15,6 \\
Hembras compradas & 6,3 & 8,9 & 9,5 & 7,6 \\
Hembras reemplazo & 43,7 & 41,1 & 40,5 & 42,4 \\
\hline
\end{tabular}

* Estrato es la unidad de producción de ovinos con base en el número de vientres: $\mathrm{E} 1=1-20 ; \mathrm{E} 2=21-50 ; \mathrm{E} 3=>50$.

No se midió la prolificidad, solamente se obtuvo el porcentaje de hembras con más de una cría. El E3 presentó $42,7 \%$ de partos con más de una cría, seguido del E2 y E1 con 41,5 y $39 \%$ respectivamente.

Los registros son sencillos, no contienen la información necesaria para realizar un análisis del rebaño tal y como se debe de llevar en una empresa, lo que no permitió obtener indicadores productivos con mayor precisión. En general un 25,6\% de productores llevan solamente registros ocasionales y de manera aislada. Por estratos el que mayor porcentaje los usó fue el E3 con $36,4 \%$, seguido del E1 y E2 con 25,5 y $21,9 \%$, respectivamente.

\section{Manejo sanitario}

Las principales enfermedades que afectan a los ovinos en los rebaños del estado son: neumonías $(37,0 \%)$, clostridios $(27,7 \%)$, carbón sintomático $(20,2 \%)$, derriengue $(9,2 \%)$ y miasis nasal $(5,8 \%)$ (Figura 4). En E3 la enfermedad más frecuente es la causada por clostridios con un $38,9 \%$, lo anterior se puede entender ya que los productores de E3 son los que presentan un mayor grado de tecnificación, utilizando con frecuencia jaulas elevadas y mayor hacinamiento de animales en sus corrales, lo cual genera una alta concentración de heces propiciando la presencia de bacterias que se diseminan en los corrales e instalaciones.

Los principales parásitos externos o ectoparásitos que atacan a los ovinos son el tábano con un 55,3\%, garrapatas $29,1 \%$ y moscas con un $15,6 \%$, esta incidencia 


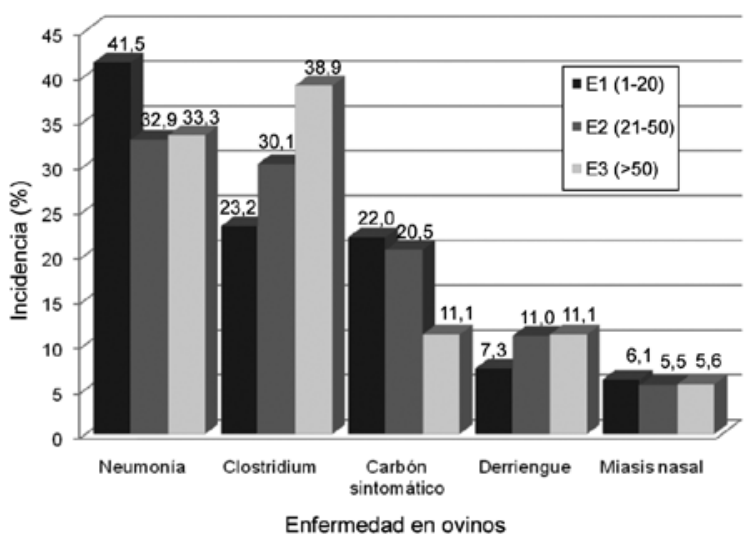

Figura 4. Enfermedades frecuentes en ovinos y porcentaje de control sanitario por estrato. Yucatán, México. 2007.

es variable en los rebaños ovinos dependiendo de las condiciones de control y prevención, así como del estado físico de potreros e instalaciones. La mayor incidencia de la garrapata se dio en $\mathrm{E} 1$, con un $31,9 \%$, debido a su bajo control. Mientras que la mayor presencia de tábanos se observó en rebaños del E3 con $60 \%$, resultado del hacinamiento de animales en los corrales de engorda. En términos generales el combate de ectoparásitos solo es realizado en el 14,4\% de los rebaños ovinos. En E1 sólo lo efectúa el 4,3\%, mientras que en E2 y E3 es de 25 y 27,3\%, respectivamente.

Los parásitos internos infestan al animal por medio de la ingestión de larvas, las cuales se desarrollan a partir de los huevos que salen junto con las heces de los animales y que contaminan los potreros, comederos y bebederos. Los resultados obtenidos muestran que los parásitos internos son causa de problemas en el rebaño, ya que en E1 y E2 este problema se manifiestó en el 23,4 y 21,9\%, respectivamente, mientras que en E3 se presentó en el 54,5\% de los rebaños. Entre los principales parásitos identificados están Oestrus, Taenias y Verme gastrointestinal y pulmonar. El 76,6 y $78,1 \%$ de los productores del E1 y E2 manifestaron que no tienen problemas de parasitosis en sus rebaños, mientras que en E3 este porcentaje desciende a 45,5\%. Este alto porcentaje encontrado en E1 y E2 se pudo atribuir a que los productores de estos estratos no realizan medidas de supervisión y muestreo de sus animales para poder determinar si tienen o no problemas con estos parásitos, aunado a esto sus periodos de desparasitación están fuera de lo recomendado (Cuadro 4).
Cuadro 4. Periodos de desparasitación realizados en ovinos (parásitos internos). Yucatán, México. 2007.

\begin{tabular}{lccc}
\hline \multirow{2}{*}{$\begin{array}{l}\text { Periodo de } \\
\text { desparasitación }\end{array}$} & \multicolumn{3}{c}{ Estrato por número de vientres* } \\
\cline { 2 - 4 } & $\mathbf{E ~ 1 ~}$ & $\mathbf{E ~ 2 ~}$ & $\mathbf{E 3}$ \\
\cline { 2 - 4 } & \multicolumn{3}{c}{)} \\
\hline Cada cuatro meses & - & 28,1 & 45,5 \\
Cada seis meses & 36,2 & 34,4 & 54,5 \\
Cada año & 63,8 & 37,5 & - \\
\hline
\end{tabular}

* Estrato es la unidad de producción de ovinos con base en el número de vientres: $\mathrm{E} 1=1-20 ; \mathrm{E} 2=21-50 ; \mathrm{E} 3=>50$.

La información disponible evidencia una variación en la frecuencia de desparasitación, la cual se explica en parte por el desconocimiento que tiene el productor de su importancia. Si el productor no identifica animales parasitados, no podrá implementar un programa de desparasitación en su rebaño.

El 68,9\% de los productores que supervisan a sus animales, realizan esta práctica en los corrales de manejo y el restante $31,1 \%$ en las zonas de pastoreo. En E1 sólo el 28\% supervisó a sus animales, mientras para el E2 y E3 fue el 62 y 90\%, respectivamente. En los estratos la supervisión fue realizada principalmente en el corral de manejo y obedece a la práctica común de concentrar a los animales después del pastoreo.

En general, los productores mencionaron tener un mayor interés en la supervisión de las borregas con cría $(45,9 \%)$ y los borregos de engorda $(29,6 \%)$. En E3, la supervisión fue en los animales de engorda (45,5\%), mientras que en E1 y E2, se orientó a las borregas con cría $(57,4$ y 43,8\%), debido a que en E3 existen mayor número de productores los cuales se dedican al engorde.

Se concluye que la supervisión de los rebaños en Yucatán, está condicionado por el estrato del productor, lo cual denota que a mayor número de vientres es mayor la supervisión de los animales y por lo tanto mejor manejo sanitario.

Los rebaños de ovinos presentan problemas de abortos durante todo el año, los cuales fluctúan dependiendo de la época. En el Cuadro 5, se observa que el mayor porcentaje de abortos se dio en la época seca, cuando existe una baja disponibilidad de alimento.

En el presente estudio, el mayor índice de mortalidad predestete $(12,2 \%)$, fue similar a los reportados 
Cuadro 5. Problemas de abortos en ovinos y su incidencia por número de vientres y época del año. Yucatán, México. 2007.

\begin{tabular}{lccc}
\hline & \multicolumn{3}{c}{ Estrato por número de vientres* } \\
& \multicolumn{3}{c}{$\mathbf{( \% )}$} \\
\cline { 2 - 4 } Concepto & $\mathbf{E 1}$ & $\mathbf{E 2}$ & $\mathbf{E 3}$ \\
\hline \% Problemas de abortos & 53,2 & 34,4 & 18,2 \\
\% Vientres con abortos & 4,95 & 3,7 & 2 \\
Época & & & \\
Sequia & 52,0 & 63,6 & 100 \\
Lluvias & 20,0 & 9,1 & - \\
Frío & 28,0 & 27,3 & - \\
\hline
\end{tabular}

* Estrato es la unidad de producción de ovinos con base en el número de vientres: $\mathrm{E} 1=1-20 ; \mathrm{E} 2=21-50 ; \mathrm{E} 3=>50$.

por Murguía et al. (2007) y Ramírez (2004) quienes encontraron en rebaños de pequeños productores, una mortalidad predestete de 10 y $11,76 \%$ respectivamente (Cuadro 6).

Cuadro 6. Mortalidad ovina según etapa fisiológica y por estrato en el estado de Yucatán, México. 2007.

\begin{tabular}{lccc}
\hline \multirow{2}{*}{ Concepto } & \multicolumn{3}{c}{ Estrato por numero de vientres* (\%) } \\
\cline { 2 - 4 } & E1 & E2 & E3 \\
\hline Predestete & 12,2 & 5,6 & 2,2 \\
Posdestete & 4,4 & 3,3 & 2,2 \\
Engorda & 2,2 & 3,3 & - \\
Hembras adultas & 2,2 & 2,2 & 2,2 \\
Sementales & 1,1 & - & - \\
\hline
\end{tabular}

* Estrato es la unidad de producción de ovinos con base en el número de vientres: $\mathrm{E} 1=1-20 ; \mathrm{E} 2=21-50 ; \mathrm{E} 3=>50$.

\section{Análisis de comercialización}

El sistema de trato o venta es reflejo en su mayoría de las condiciones de ventaja de conocimientos del mercado que tiene cada productor, como se observa sólo el 16,3\% de los ovinocultores tratan o venden sus animales a través de alguna asociación ganadera, mientras que el restante $83,7 \%$ lo realizan directamente en la unidad de producción (Cuadro 7). En el E1
Cuadro 7. Lugar donde se realiza o trata la venta de animales ovinos. Yucatán, México. 2007.

\begin{tabular}{lcccc}
\hline Lugar & \multicolumn{4}{c}{ Estrato por número de vientres* $(\%)$} \\
\cline { 2 - 5 } & E1 & E2 & E3 & Total \\
\hline $\begin{array}{l}\text { En la unidad de } \\
\text { producción }\end{array}$ & 100,0 & 87,5 & 63,6 & 83,7 \\
$\begin{array}{l}\text { A través de la Aso- } \\
\text { ciación }\end{array}$ & - & & & \\
\hline
\end{tabular}

* Estrato es la unidad de producción de ovinos con base en el número de vientres: $\mathrm{E} 1=1-20 ; \mathrm{E} 2=21-50 ; \mathrm{E} 3=>50$.

el $100 \%$ de los productores realiza o trata sus ventas en sus ranchos; esta forma tiene una desventaja de precio de su producto, ya que bajo estas condiciones los precios presentan una mayor variabilidad que si el productor vendiese a través de la asociación, ya que en ésta existen precios predeterminados por las condiciones del mercado y no por la voluntad de los intermediarios. Contrario a E1, los productores de E3, en su mayoría $(63,6 \%)$ realizan sus ventas a través de la asociación.

Al respecto, Góngora y Franco (1997), hacen referencia que existe en el estado de Yucatán una falta de organización de pequeños productores de ovinos, lo cual induce a vender, en la mayoría de los casos, al mejor postor o de acuerdo a sus necesidades en su rancho.

Con relación a los criterios que tienen los productores para decidir el momento de la venta de sus animales, se observó que el principal de éstos se asocia con el peso de sus animales al mercado $(68,84 \%)$, seguido con menor importancia está la necesidad de dinero, sobrepoblación de su hato, insistencia del comprador y la falta de alimento para su rebaño. Estos criterios variaron dependiendo el estrato. En el E1 la necesidad de dinero fue el principal factor en la decisión de venta (Cuadro 8), ésto se entiende por qué sus rebaños están conformados hasta con 20 animales, representan un ahorro o alternativa de consumo de la familia. Por su parte, en el E3 el criterio de sobrepoblación se relaciona con la falta de alimento disponible para el rebaño en las épocas de escasez. En E1 los compradores están representados por intermediarios o engordadores, los cuales buscan un bajo precio de adquisición de animales en este tipo de productores. 
Cuadro 8. Criterios adoptados por el productor para decidir el momento de la venta de sus animales ovinos. Yucatán, México. 2007.

\begin{tabular}{|c|c|c|c|c|}
\hline \multirow[t]{2}{*}{ Concepto } & \multicolumn{4}{|c|}{$\begin{array}{c}\text { Estrato por número de vientres* } \\
(\%)\end{array}$} \\
\hline & E1 & E2 & $\mathbf{E 3}$ & Total \\
\hline Peso al mercado & 52,6 & 80,8 & 71,8 & 68,4 \\
\hline Necesidad de dinero & 37,9 & 15,6 & 6,4 & 20,0 \\
\hline Sobrepoblación & - & 1,3 & 18,2 & 6,5 \\
\hline $\begin{array}{l}\text { Insistencia del com- } \\
\text { prador }\end{array}$ & 6,6 & 2,3 & - & 3,0 \\
\hline Falta de alimento & 3,0 & - & 3,6 & 2,2 \\
\hline
\end{tabular}

* Estrato es la unidad de producción de ovinos con base en el número de vientres: E1=1-20; E2=21-50; E3= >50.

Con respecto al comportamiento de los precios durante el año, en algunos casos y dependiendo de la situación del mercado y tipo de producción de la actividad, se tienen meses en los cuales es favorable la venta de los animales. En general, en el estado de Yucatán, el precio de la carne ovina presenta cierta estabilidad, aunque con tendencias al alza por la amplia demanda del producto. El precio del ovino en pie y canal se ha incrementado en forma lenta pero sostenida, pasando de $\$ 5,06$ y 10,42 en 1990 a $\$ 10,52$ y 20,74 en 1996. En el 2000, el precio se incrementó a \$ 17,51 y $31,59 \mathrm{~kg}$ en pie y canal. Actualmente los precios son de $\$ 22,00$ y $\$ 38,00$ respectivamente (SIAP-SAGARPA 2007). Esta tendencia que han seguido los precios supone que la actividad está influenciada en forma positiva por esta variable, la cual, según el nivel de costos promedios, determina la rentabilidad y la dinámica de la ovinocultura.

Un estudio realizado por Góngora y Franco (1997) en el estado de Yucatán, concluye que existe una variación en el precio de "venta en pie" dado porque la comercialización de ovinos está en manos de intermediarios, siendo el margen de comercialización favorable a éstos en un $75 \%$. Aun cuando en la actualidad el precio del ovino se considera como estable y creciente, en los mercados locales este precio es dinámico debido a que los canales de comercialización interviene más de un actor en el proceso de compra venta, siendo el productor el que obtiene los menores ingresos.

El porcentaje de productores los cuales mencionan los meses de variación de precio del producto ya sea al alza, baja o sin variación se muestra en la Figura 5. Esta información se relaciona con los meses de mayor y menor venta (Figura 7), dicha variación de precios se puede estar dando por la gran variedad de compradores de ovinos presentes en el estado así como por el destino y finalidad en el uso del animal.

En el Cuadro 9, se muestra la proporción de ventas de animales, realizadas a los diferentes tipos de compradores. Tanto los comisionistas y los mayoristas de animales en pie, sumaron casi el 51,9\% de las ventas, coincidiendo con lo encontrado por Góngora y Franco (1997).

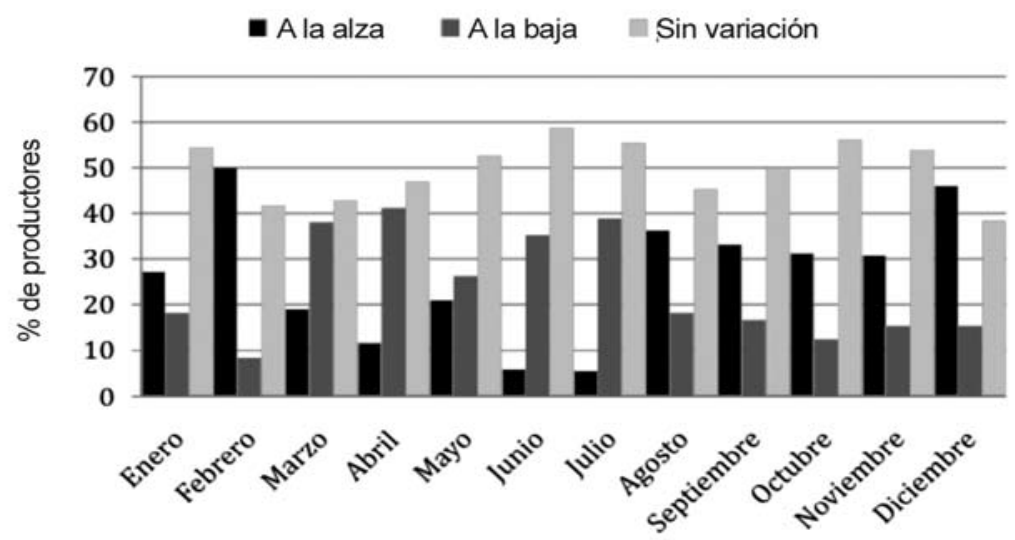

Figura 5. Porcentaje de productores que identifican variación mensual en el precio del ovino en pie en el estado de Yucatán, México 2007. 
Cuadro 9. Ventas de ovinos realizadas a las diferentes tipos de compradores. Yucatán, México. 2007.

\begin{tabular}{lcccc}
\hline \multirow{2}{*}{ Concepto } & \multicolumn{4}{c}{ Estrato por número de vientres* $(\%)$} \\
\cline { 2 - 5 } & E1 & E2 & E3 & Total \\
\hline $\begin{array}{l}\text { Comisionista } \\
\text { animales }\end{array}$ & 18,9 & 5,8 & 3,3 & 28,0 \\
$\begin{array}{l}\text { Mayorista de ani- } \\
\text { males en pie }\end{array}$ & 11,9 & 5,3 & 6,7 & 23,9 \\
$\begin{array}{l}\text { Otro productor } \\
\text { En la localidad }\end{array}$ & 10,2 & 3,1 & 1,3 & 14,7 \\
Abastecedor & 5,6 & 8,0 & 0,2 & 13,8 \\
Asociación & 2,9 & 5,9 & - & 8,8 \\
Restaurantes & 1,1 & 6,9 & 0,7 & 8,7 \\
Procesador o car- & & 0,3 & - & 1,7 \\
nicero & 0,3 & 0,2 & - & 0,6 \\
\hline
\end{tabular}

* Estrato es la unidad de producción de ovinos con base en el número de vientres: $\mathrm{E} 1=1-20 ; \mathrm{E} 2=21-50 ; \mathrm{E} 3=>50$.

\section{Estudio socioeconómico}

Para este estudio se analizó las medias de la productividad contra aquellas variables las cuales pueda existir una relación, éstas son:

\section{1) Escolaridad del productor (años cursados)}

La variable de escolaridad o los años cursados por el productor, no estuvo relacionada con la productividad del rebaño, así la mayor productividad se obtuvo en productores de escolaridad media con estudios de secundaria. Entre la escolaridad del productor y la productividad del rebaño no existió significancia para ninguna de sus medias analizadas (Cuadro 10). El 60\% de los ovinocultores del estado de Yucatán, cuentan con sólo el nivel básico de educación.

\section{2) Años de experiencia del productor}

De forma similar a lo obtenido con la variable de escolaridad, no existió diferencia significativa para la variable años de experiencia del productor en la productividad del rebaño (Cuadro 11).

Existen productores los cuales se encuentran integrados a la ovinocultura con visión empresarial y
Cuadro 10. Comparación de medias entra escolaridad del productor y productividad del rebaño de ovinos. Yucatán, México. 2007.

\begin{tabular}{lccc}
\hline $\begin{array}{c}\text { Escolaridad } \\
(\text { año })\end{array}$ & $\begin{array}{c}\text { Media } \\
(\mathbf{X})\end{array}$ & $\begin{array}{c}\text { Número de } \\
\text { productores }\end{array}$ & $\begin{array}{c}\text { Desviación } \\
\text { estándar }\end{array}$ \\
\hline $0-6$ & 48,21 & 54 & 21,41 \\
$7-9$ & 61,62 & 17 & 24,76 \\
$10-12$ & 53,57 & 15 & 27,02 \\
Más de 12 & 51,05 & 4 & 13,55 \\
\hline
\end{tabular}

Cuadro 11. Comparación de medias entre años de experiencia del productor y productividad del rebaño de ovinos. 2007.

\begin{tabular}{lccc}
\hline $\begin{array}{c}\text { Antigüedad } \\
\text { (año) }\end{array}$ & $\begin{array}{c}\text { Media } \\
(\mathbf{X})\end{array}$ & $\begin{array}{c}\text { Número de } \\
\text { productores }\end{array}$ & $\begin{array}{c}\text { Desviación } \\
\text { estándar }\end{array}$ \\
\hline $0-5$ & 51,52 & 7 & 21,37 \\
$6-10$ & 56,75 & 30 & 20,07 \\
$11-15$ & 50,56 & 31 & 25,62 \\
Más de 15 & 42,33 & 22 & 23,83 \\
\hline
\end{tabular}

con cierto nivel de aplicación de tecnología, y los productores los cuales conllevan la actividad de manera tradicional con un fin de apoyo económico. El primer tipo de productor se encuentra en la actividad de 15 años a la fecha, presentando un $41,1 \%$ de los productores a nivel estado, mientras que el segundo grupo de productores su antigüedad es mayor a los 15 años, siendo éstos el 58,9\%.

\section{3) Superficie utilizada}

La variable superficie presentó diferencias significativas, los productores que poseen mayor número de hectáreas en uso para la ovinocultura, presentaron mayor productividad en su rebaño. La productividad de los productores que poseen de 15 a 30 ha y los de más de 30 son significativamente diferentes a los que cuentan con menor número de ha ( 1 a 5 ). Se puede dar por entendido que a mayor número de hectáreas disponibles para la producción ovina, existirá una mayor área de pastoreo lo cual hará más productivo al rebaño. Esta tendencia es positiva siempre y cuando las hectáreas disponibles se utilicen de manera eficiente (Cuadro 12). 
Cuadro 12. Comparación de medias entre la superficie utilizada y la productividad del rebaño de ovinos. Yucatán, México. 2007.

\begin{tabular}{lccc}
\hline $\begin{array}{c}\text { Superficie } \\
\text { (ha) }\end{array}$ & $\begin{array}{c}\text { Media } \\
(\mathbf{X})\end{array}$ & $\begin{array}{c}\text { Número de } \\
\text { productores }\end{array}$ & $\begin{array}{c}\text { Desviación } \\
\text { estándar }\end{array}$ \\
\hline $0-5$ & $32,95 \mathrm{a}^{*}$ & 17 & 10,41 \\
$6-15$ & $48,63 \mathrm{~b}$ & 36 & 17,61 \\
$15-30$ & $55,05 \mathrm{ac}$ & 25 & 24,29 \\
Más de 30 & $64,05 \mathrm{ac}$ & 12 & 19,44 \\
\hline
\end{tabular}

* Medias diferentes en la misma columna son diferentes $(\mathrm{P} \leq 0,05)$.

\section{4) Estrato de productores}

El estrato del productor se determinó por el número de vientres presentes en el rebaño, las medias de productividad presentan diferencias significativas con respecto a su estrato, a mayor número de vientres el rebaño existirá mayor productividad (Cuadro 13).

Cuadro 13. Comparación de medias entre estrato del productor y productividad del rebaño de ovinos. Yucatán, México. 2007.

\begin{tabular}{lccc}
\hline $\begin{array}{l}\text { Estrato } \\
\text { (n. de vientres) }\end{array}$ & $\begin{array}{c}\text { Media } \\
\text { (X) }\end{array}$ & $\begin{array}{c}\text { Número de } \\
\text { productores }\end{array}$ & $\begin{array}{c}\text { Desviación } \\
\text { estandar }\end{array}$ \\
\hline E1 0-20 & $48,35 \mathrm{a}^{*}$ & 47 & 22,29 \\
E2 21-50 & $52,37 \mathrm{~b}$ & 32 & 23,81 \\
E3 más de 50 & $69,12 \mathrm{ac}$ & 11 & 18,19 \\
\hline
\end{tabular}

* Medias diferentes en la misma columna son diferentes $(\mathrm{P} \leq 0,05)$.

\section{Costos de producción}

El costo de producción de la carne de ovino en pie se componen prioritariamente por los costos variables (CV), los cuales integran el 66,9\% del costo total, dentro de estos los principales componentes del valor de los insumos corresponde a la mano de obra y alimentación con un 23,7 y 21,9\% respectivamente, seguidos de la utilización de herbicidas, electricidad, combustible y el mantenimiento de la infraestructura y equipo con 5,$1 ; 4,6 ; 4,1$ y $3,1 \%$, respectivamente. El rubro que abarca menor costo de producción son los relacionados con el manejo sanitario del rebaño los cuales comprenden, desparasitantes, vacunas y medicamentos para el control de enfermedades, sumando un 4,0\% (Cuadro 14).

El cálculo de mano de obra fue del 44,3\%, la cual comprende encargados y pastores del rebaño, mano de obra eventual $(13,9 \%)$ en la cual se encuentran los jornales contratados y por última la mano de obra familiar (41,8\%); ésta última no es pagada en efectivo; ya que, se considera el reembolso por concepto de venta de animales; sin embargo, para los fines de este estudio sí fue estimada.

El costo de producción que abarca el alimento fue bajo, considerando que este costo asciende a más del $50 \%$ del costo de producción animal, una de las formas de explicar este bajo costo para la alimentación ovina en el estado de Yucatán, es la alta dependencia de áreas de pastoreo ya sea de especias introducidas o en agostaderos, en este sentido el $21,9 \%$ del costo considerado de alimentación abarcó solamente el alimento balanceado comercial proporcionado al rebaño.

En cuanto a los costos fijos (CF) éstos abarcan el $33,5 \%$ del total de la producción, siendo de interés por pie de cría el que cubrió un mayor porcentaje $(10,1 \%)$, seguido del interés de inversión de infraestructura $(8,3 \%)$. El comportamiento del costo de interés por pie de cría por estrato fue creciente (E1, 6,5\%, E1, 10,2\% y E3 $13,5 \%$ ) ya que este interés es imputado directamente al número de vientres y sementales presentes en el rebaño, esta tendencia no se refleja de igual forma para el costo por interés de inversión de infraestructura (E1, 9,4\%, E1, 7,7\% y E3 7,8\%), debido a que la tendencia por tamaño de explotación es de disminución cuando éste aumenta, aspecto que ocurrió al pasar de E1 $(9,4 \%)$ a E2 $(7,7 \%)$, pero no cuando se pasó de E2 a E3 $(7,8 \%)$. El valor por este concepto, disminuyó a medida que aumentó el tamaño del rebaño.

La depreciación de equipo e instalaciones comprenden un $15,1 \%$ del costo total (CT), presentando un comportamiento real, al ser mayor este porcentaje $(17,1 \%)$ en E1, mientras que en E2 y E3 fue de 14 y $14,2 \%$ respectivamente, lo cual indica una subutilización de maquinaria y equipo en E1 (Cuadro 14). 
Cuadro 14. Estructura porcentual de costos totales en la producción de ovinos. Yucatán. México. 2007.

\begin{tabular}{lcccc}
\hline \multirow{2}{*}{ Concepto } & \multicolumn{4}{c}{$\begin{array}{c}\text { Estrato por número de vien- } \\
\text { tres * }(\%)\end{array}$} \\
\cline { 2 - 5 } & E1 & E2 & E3 & Total \\
\hline Costo variable & 66,9 & 68,1 & 64,6 & 66,5 \\
$\quad$ Alimentación & 20,3 & 20,7 & 24,9 & 21,9 \\
$\quad$ Desparasitantes & 1,3 & 1,0 & 0,8 & 1,0 \\
$\quad$ Vacunas & 2,2 & 1,5 & 1,2 & 1,6 \\
Control de enfermeda- & 1,8 & 1,2 & 1,0 & 1,3 \\
des varias & & & & \\
$\quad$ Herbicidas & 6,2 & 5,0 & 4,2 & 5,1 \\
$\quad$ Mano de obra & 22,4 & 26,4 & 22,4 & 23,7 \\
$\quad$ Energía eléctrica & 6,7 & 4,0 & 3,1 & 4,6 \\
Mantenimiento de infra. & 2,6 & 3,4 & 3,3 & 3,1 \\
y equipo & & & & \\
Combustibles y lubri- & 3,6 & 5,0 & 3,7 & 4,1 \\
cantes & & & & \\
& & & & \\
Costo fijo & 33,1 & 31,9 & 35,4 & 33,5 \\
Depreciación de equipo & 9,0 & 6,9 & 8,0 & 8,0 \\
$\begin{array}{l}\text { Depreciación de insta- } \\
\text { laciones }\end{array}$ & 8,2 & 7,1 & 6,2 & 7,2 \\
$\begin{array}{l}\text { Interés / inversión } \\
\text { de cría }\end{array}$ & & & & \\
Interés / inversión de in- & 6,5 & 10,2 & 13,5 & 10,1 \\
fraestructura & 9,4 & 7,7 & 7,8 & 8,3 \\
\hline & & & & \\
\hline
\end{tabular}

* Estrato es la unidad de producción de ovinos con base en el número de vientres: $\mathrm{E} 1=1-20 ; \mathrm{E} 2=21-50 ; \mathrm{E} 3=>50$.

\section{Ingreso por venta de animales}

El ingreso medio por kilogramo de carne producido varía según el estrato del productor, el sector productivo y la forma de integración del mercado correspondiente. El ingreso se compone de dos variables principales: el precio y la cantidad vendida.

\section{Precio de venta al mercado}

El precio del ovino al mercado para el cálculo de ingresos se estimó por medio del precio ponderado en los estratos. Existió un incremento en la venta, debido a factores como: lugar de venta, criterios, relación de compra venta e imposición del precio
De igual forma el incremento de los precios se debe a la estructura de ventas que presenta cada estrato y a la calidad de sus animales con peso al mercado, cuyo precio fue mayor en el E3 de $18,9 \$ / \mathrm{kg}$ y decreciente para los restantes estratos. El bajo precio de los animales de E1 $(15,8 \$ / \mathrm{kg})$ se presentó en las diferentes etapas de venta de los animales (Figura 6).

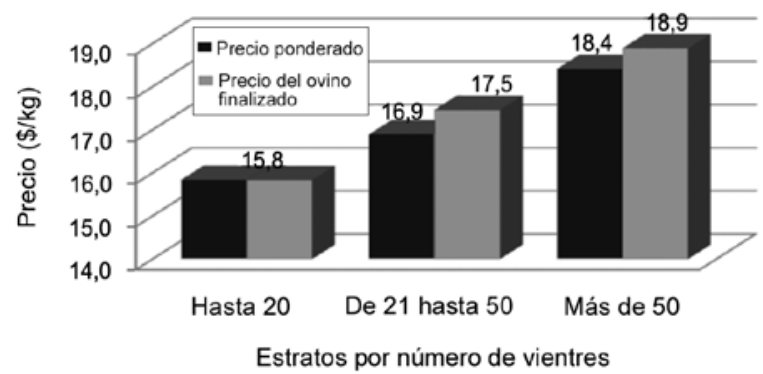

Figura 6. Precios de la carne ovina en pie. Yucatán, México. 2007.

\section{Estructura de ingreso por ventas}

Para determinar el ingreso por las ventas, se consideró tanto los $\mathrm{kg}$ de carne producidos para la venta, el cual se define como el número y clase de ganado contabilizados al principio y al final del año y del valor monetario promedio de cada etapa durante el año; pudiendo ser este valor positivo o negativo (Wadsworth 1997). La producción de carne por vientre al año consideró los $\mathrm{kg}$ totales de carne producidos entre el número de vientres en producción. El precio promedio global de venta se estimó con base al cociente del ingreso total (ingreso por venta, más cambio de inventario) entre el total de kilogramos producidos. Esto es debido, a que no se vendieron un solo tipo de animales a un mismo precio; por lo que hay una variación tanto en el precio como en el tipo de animales; y a su vez, se considera el valor del cambio de inventario tanto en dinero como en peso.

Un aspecto estrechamente relacionado con el desempeño y el objetivo de la empresa es su estructura de ingresos por ventas, ya que ésta es resultado de la conjugación de los elementos técnicos, de la disponibilidad de recursos productivos y de la capacidad de administración que orienta el empleo de los dos primeros factores. 
La estructura porcentual de ingresos por venta en el estado de Yucatán se encuentra orientada a la venta de animales con peso al mercado $(54,2 \%)$, seguido de la venta de destetes $(21,5 \%)$. Esta alta orientación de ventas de animales con peso al mercado se ve reflejada en los tres estratos (Cuadro 15).

Cuadro 15. Estructura porcentual del ingreso por ventas de ovinos. Yucatán, México. 2007.

\begin{tabular}{lcccc}
\hline \multirow{2}{*}{\multicolumn{1}{c}{ Venta }} & \multicolumn{4}{c}{ Estrato por número de vientres* $(\%)$} \\
\cline { 2 - 5 } & E1 & E2 & E3 & Total \\
\hline Destetes & 21,0 & 29,6 & 13,9 & 21,5 \\
Finalizados & 54,8 & 49,8 & 57,9 & 54,2 \\
Sementales & 3,1 & 1,2 & 4,5 & 3,0 \\
Vientres & 2,4 & 1,6 & 16,0 & 6,7 \\
Hembra desecho & 16,8 & 15,8 & 7,7 & 13,4 \\
Machos desecho & 1,8 & 2,0 & 0,0 & 1,3 \\
Venta total & 100,0 & 100,0 & 100,0 & 100,0 \\
\hline
\end{tabular}

* Estrato es la unidad de producción de ovinos con base en el número de vientres: $\mathrm{E} 1=1-20 ; \mathrm{E} 2=21-50 ; \mathrm{E} 3=>50$.

\section{Rentabilidad de los estratos de producción}

La rentabilidad de la producción de carne ovina en el estado de Yucatán presentó variaciones y pérdidas dependiendo del estrato del productor. El valor promedio es positivo sólo para las explotaciones de E3 y cuya magnitud resultó de 2,8 pesos por cada kilogramo de carne en pie producido en el año 2007 (Figura 7). Por su parte, la rentabilidad negativa (pérdida absoluta) que en promedio experimentaron las explotaciones de E1 y E2 fue ocasionada por su menor precio ponderado de venta obtenido y a la existencia de un mayor costo relativo por kilogramo de carne en pie.

De igual forma se calculó la rentabilidad solamente sobre los $\mathrm{CV}$, cuando a nivel contable no se recupera el CF, tal y como suele suceder en las explotaciones donde sólo se considera el CV, el indicador de rentabilidad resulta positivo o de mayor magnitud al real (Figura 7). Este caso reviste una importancia significativa en las explotaciones de E1 y E2, donde la rentabilidad real es negativa (el productor no recupera el capital invertido), pero según esta forma de estimación por omisión del CF, resulta positiva. Así, ante un estímulo irreal se continuará en la actividad

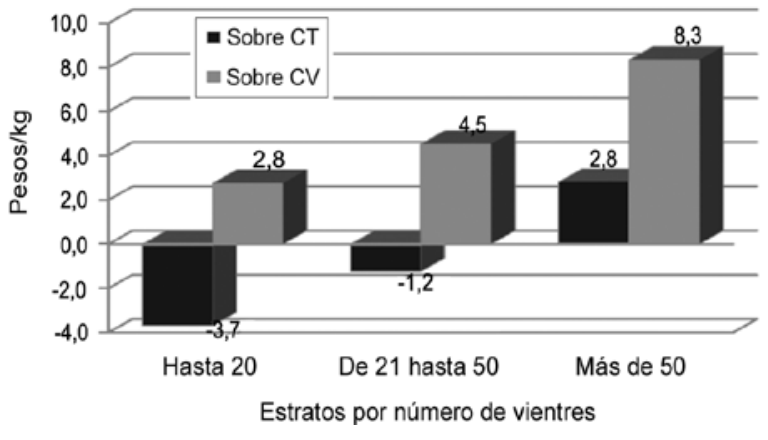

Figura 7. Rentabilidad de la producción de ovino en pie. Yucatán, México. 2007.

hasta que la falta de liquidez para la reposición de activos, induzca, en primer lugar, a la pérdida de productividad y dinamismo de la explotación y, por último, a la inactividad y/o venta.

El cambio hacia una actitud de tipo empresarial es indispensable en la producción de cualquier especie animal o rubro agropecuario, con base en objetivos y estrategias bien definidas a corto, mediano y largo plazo, que determinen el manejo técnico y económico. Es indispensable llevar registros de producción y contables, para el control y análisis, tanto de los datos productivos como económicos, que permitan fijar objetivos y tomar decisiones que garanticen mayores ganancias.

\section{LITERATURA CITADA}

CONAGUA (Comisión Nacional del Agua). 2008. Organismo de cuenca península de Yucatán (en línea). Dirección Técnica, Jefatura de proyecto de aguas superficiales y meteorología. Mérida Yucatán, México. Centro regional de pronóstico meteorológico. Consultado 1 ene. 2008. Disponible en http://www.conagua.gob. $\mathrm{mx} / \mathrm{OCPY} 07 /$ Contenido/Documentos/BolEst.pdf

Franco, CA; Esquivel, H; Sarmiento, L; Moncada, E; Ríos, G. 1992. Caracterización de la ovinocultura en la región noreste del estado de Yucatán. In: Reunión Nacional de Investigación Pecuaria, Chihuahua, México. p. 368.

Góngora, S; Franco, C. 1997. Análisis de la comercialización ovina en el estado de Yucatán. In: Memoria III Seminario. La investigación socioeconómica en el estado de Yucatán y su impacto sobre el sector agropecuario. Yucatán, México. p. 150.

INEGI (Instituto Nacional de Estadística y Geografía). 2006. Sistema para la consulta del anuario estadístico del 
estado de Yucatán (en línea). Edición 2006. México. Consultado 1 dic. 2006. Disponible en http://www. inegi.gob.mx/est/contenidos/espanol/sistemas/aee06/ estatal/yuc/index.htm

Kay, RD. 1990. Administración agrícola y ganadera: planeación control e implementación. 7 ed. México, D.F. McGraw-Hill. 432 p.

Magaña, MM; Sierra, VA; Martínez, A. 2000. Caracterización del sistema productivo de ovino Pelibuey en Yucatán. In: XL Congreso Nacional de Investigación y Desarrollo Tecnológico Agropecuario, Villa Ocuilzapotlan, Tabasco, México. 207 p.

Murguía, O, M, Rojas R, O, Solís C, J. 2007. Status sanitario de rebaños ovinos de Yucatán. Prácticas preventivas. In: Memoria XLIII Reunión Nacional de Investigación Pecuaria Sinaloa, México. 2007. p. 56.
Murray, RS; Larry, JS. 2001. Estadística. Mc Graw Hill Interamericano, Impreso en México 2001.PÁGINAS??

Ramírez M, A. 2004. Rentabilidad de la producción ovina en Yucatán, tesis de maestría. Instituto Tecnológico de Conkal Yucatán, México. p. 125.

SIAP-SAGARPA. 2006. Población ganadera "ovinos" (en línea). México. Consultado 1 dic. 2006. Disponible en http://www.siap.gob.mx/integracion/estadisticabasica/ pecuario/ar_pobgan.php.

SIAP-SAGARPA. 2007. Precios del ovino en pie y canal (en línea). México. Consulta 16 oct. 2006. Disponible en http://www.siap.gob.mx/

Wadsword, J. 1997. Análisis de sistemas de producción animal. Tomo 2. In: Las herramientas básicas. Estudio FAO Producción y Sanidad Animal 140/2. p. 123. 